Classification

Physics Abstracts

76.30F

\title{
ESR in a 1D-system with a ferromagnetic coupling
}

\author{
S. Poertadji, G. Ablart, J. Pescia \\ Laboratoire de Magnétisme et d'Electronique Quantique, \\ Université Paul Sabatier, 39, Allée Jules-Guesde, 31062 Toulouse Cedex, France
}

\author{
S. Clément and A. Cheik-Rouhou \\ Institut d'Electronique Fondamentale (*), \\ Bâtiment 220, Université de Paris XI, 91405 Orsay Cedex, France
}

(Reçu le 6 avril 1983, révisé le 10 mai, accepté le 16 mai 1983)

\begin{abstract}
Résumé. - Nous avons mesuré les dépendances angulaires de la largeur de raie de RPE et du facteur $g$ en bande $\mathrm{X}$ et à la température ambiante dans le trichlorure cuivrique de cyclo-hexyl-ammonium (ou CHAC). Nous avons déterminé également la dépendance de la largeur de raie avec la température entre 30 et $300 \mathrm{~K}$.
\end{abstract}

\begin{abstract}
We have measured the angular dependences of the ESR linewidth and $g$-factor at $\mathrm{X}$-band and room temperature in cyclo-hexyl-ammonium-trichlorocuprate (II) (CHAC). The temperature dependence of the linewidth between 30 and $300 \mathrm{~K}$ has also been determined.
\end{abstract}

\section{Introduction.}

The magnetism of one-dimensional (1D) systems has been mainly investigated in manganese compounds presenting an antiferromagnetic coupling such as tetramethyl manganese chloride (TMMC) and cesium manganese chloride (CMC) [1-3]. TMMC can be considered, to date, as the best 1D-system available for experiment. Recent progress in material chemistry has allowed the synthesis of linear Ising-Heisenberg systems with a ferromagnetic coupling [4]. The cyclohexyl-ammonium-trichlorocuprate (II) $\left(\left(\mathrm{C}_{6} \mathrm{H}_{11} \mathrm{NH}_{3}\right) \mathrm{CuCl}_{3}\right)$, usually termed "CHAC " is a typical example of these materials $[5,6]$. The experimental data concerning the magnetic susceptibility $[5,7]$ and the specific heat $[6,8]$ indicate that it is very close to the theoretical onedimensional model, because of a strong interchain exchange and $\left(J_{0} \sim 50 \mathrm{~K}\right)$ and a weak interchain exchange $\left(J^{\prime} \sim 10^{-3} \times J_{0}\right)$. It shows a small anisotropic exchange so that the Hamiltonian of the symmetrical exchange can be expressed as :

$$
H_{\mathrm{e}}^{\mathrm{s}}=-\sum_{i, j} J_{\perp}\left(S_{i}^{x} S_{j}^{x}+S_{i}^{y} S_{j}^{y}\right)+J_{\|} S_{i}^{z} S_{j}^{z}
$$

$\left(^{*}\right)$ Laboratoire associé au C.N.R.S. 
With this type of Hamiltonian two extreme cases may be considered : (i) $J_{\perp} / J_{\|}=1$ which leads to a Heisenberg system, (ii) $J_{\perp} / J_{\|}=0$ which corresponds to an Ising system. In CHAC, $J_{\perp} / J_{\|}=0.99$ [7], it shows a strong Heisenberg interaction and a very weak Ising one.

In this communication, we present experimental data concerning Electron Spin Resonance (ESR) at X-band in CHAC. We have measured the linewidth in the temperature region between 20 and $300 \mathrm{~K}$. Furthermore, we have determined, at room temperature, the dependences of the linewidth and the $g$-factor with the angle $\theta$ between the crystal $c$-axis and the magnetic field $\mathbf{H}_{0}$. Finally, we discuss our data taking into account the various interactions in the sample.

\section{Sample.}

The crystal was grown from an equimolar solution of ammonium cyclohexyl chloride and anhydrous copper chloride (II) in 1-propanol. The crystallographic structure of CHAC has been determined by Groenendijk et al. [7]. The $\mathrm{CuCl}_{3}^{-}$-ions are disposed in chains parallel to the $c$-axis. They are bound by the hydrogen bonds in the $(b-c)$ plane, through the $\mathrm{NH}_{3}^{+}$-groups.

At room temperature the structure is orthorhombic with $a=19.441 \AA, b=8.549 \AA$ and $c=6.190 \AA$ [7]. The apical $\mathrm{Cu}-\mathrm{Cl}$ (1) bond is longer than the equatorial $\mathrm{Cu}-\mathrm{Cl}$ (2) or $\mathrm{Cu}-\mathrm{Cl}$ (3) one. The hydrogen bond produces a weak distorsion which displaces the $\mathrm{Cu}^{2+}$-ion out of the equatorial plane constituted by the four basal $\mathrm{Cl}^{-}$-ions, towards the apical chlorine. $\mathrm{A} \mathrm{Cu}-\mathrm{Cl}-\mathrm{Cu}$, bridge appears in the chain between two adjacent copper ions with an angle of $86^{\circ}$ between the two $\mathrm{Cu}-\mathrm{Cl}$ bonds. The bridging angle of less than $90^{\circ}$ leads to a strong ferromagnetic coupling within each chain [7]. The distance between two adjacent copper atoms is $3.138 \AA$ along the $c$-axis while it is $8.549 \AA$ along the $b$-axis and $10 \AA$ along the $a$-axis. Furthermore, the magnetic ordering occurs below $T_{\mathrm{c}}=2.18 \mathrm{~K}[6,7]$.

\section{The experimental arrangement.}

We have measured the linewidth with an ESR spectrometer operating at the frequency of $9.4 \mathrm{GHz}$. The sample, of size $3 \times 2 \times 1.5 \mathrm{~mm}^{3}$, was placed within the resonant cavity. A goniometric device allowed rotations in a plane including the magnetic field $\mathbf{H}_{0}$. The angle $\theta$ between $H_{0}$ and the $c$-axis was measured with an accuracy of $\pm 0.5^{\circ}$. The temperature was varied from 20 to $300 \mathrm{~K}$ using an "OXFORD instruments » cryostat and it was measured by a thermocouple fixed close to the sample.

\section{Experimental data.}

First, we have measured the angular dependence of the $g$-factor in the $(a, c)$ and $(b, c)$ planes. The data are shown in figure 1 . They are well described by the following equation, for $H_{0}$ in the $(\alpha, c)$ plane and at $300 \mathrm{~K}$ :

$$
g(\theta)=\left(g_{c}^{2} \cos ^{2} \theta+g_{\alpha}^{2} \sin ^{2} \theta\right)^{1 / 2},
$$

where $\alpha$ is the $a$ or $b$-axis, with :

$$
g_{a}=2.108 \pm 0.001 ; \quad g_{b}=2.185 \pm 0.003 ; \quad g_{c}=2.129 \pm 0.003
$$

The $g_{a}$ and $g_{c}$-values are in good agreement with those found by Groenendijk et al. at $80 \mathrm{~K}$ [7], but a difference occurs for the $g_{b}$-value. It is due to different temperature dependences. In effect, when the temperature is lowered below $300 \mathrm{~K}, g_{b}$ increases continuously while $g_{a}$ and $g_{c}$ decrease below 70 and $30 \mathrm{~K}$ respectively.

The angular variations of the linewidth observed at room temperature are shown in figure 2 . The measurements have been taken in the $(a, c)$ and $(b, c)$ planes. They are well fitted by the 


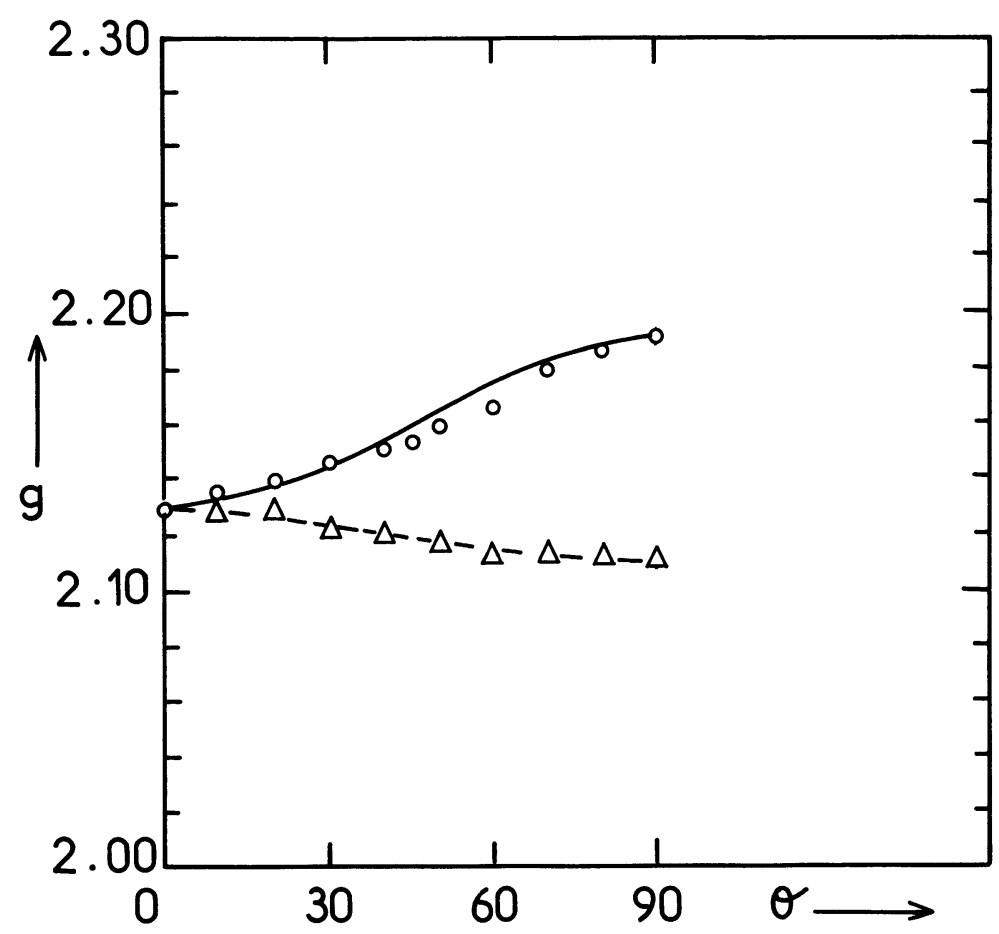

Fig. 1. - The $g$-factor in CHAC versus the angle $\theta$ between the $c$-axis and the magnetic field $\boldsymbol{H}_{0}$. The temperature is $300 \mathrm{~K}$ and the spectrometer operates at X-band. The circles and the triangles correspond to the magnetic field in the $(b, c)$ and $(a, c)$ planes respectively. The solid line has been drawn from the computation of $\left(g_{c}^{2} \cos ^{2} \theta+g_{b}^{2} \sin ^{2} \theta\right)$ and the dotted curve from that of $\left(g_{c}^{2} \cos ^{2} \theta+g_{a}^{2} \sin ^{2} \theta\right)$ with $g_{a}=2.108 \pm 0.001$; $g_{b}=2.185 \pm 0.003$ and $g_{c}=2.129 \pm 0.005$.

following expressions, found from an adjustment by the mean square method :

$$
\begin{array}{ll}
\text { in the }(a, c) \text { plane } & \Delta H(\theta)=788-413 \cos ^{2} \theta \\
\text { in the }(b, c) \text { plane } & \Delta H(\theta)=860-485 \cos ^{2} \theta .
\end{array}
$$

In figure 3 we have drawn the variation of the linewidth with the temperature in the range from 20 to $300 \mathrm{~K}$ for the orientations $\theta=0^{\circ}$ and $\theta=90^{\circ}$, with $H_{0}$ in the $(a, c)$ plane.

\section{Discussion.}

The angular anisotropy and the temperature dependence of the linewidth in CHAC are very different from those usually observed in 1D-systems. In TMMC for instance, the linewidth varies as $\left|1-3 \cos ^{2} \theta\right|^{4 / 3}$ and presents a marked minimum at the magic angle $\theta=55^{\circ}$, while in CHAC it increases when $\theta$ varies from zero to $90^{\circ}$, in the two planes studied. Another difference concerns the lineshape. In TMMC, the ESR line shows a form intermediate between a Gaussian and a Lorentzian, except at the magic angle where it is purely Lorentzian. In contrast, the lineshape of CHAC remains approximately Lorentzian, whatever the orientation.

In TMMC the linewidth is essentially determined by the dipolar Hamiltonian $H_{\mathrm{D}}$, presenting an axial symmetry with respect to the chain axis. $H_{\mathrm{D}}$ is also responsible for the breakdown of diffusing modes along the chains, which gives a non-Lorentzian lineshape. Thus, it is clear that 


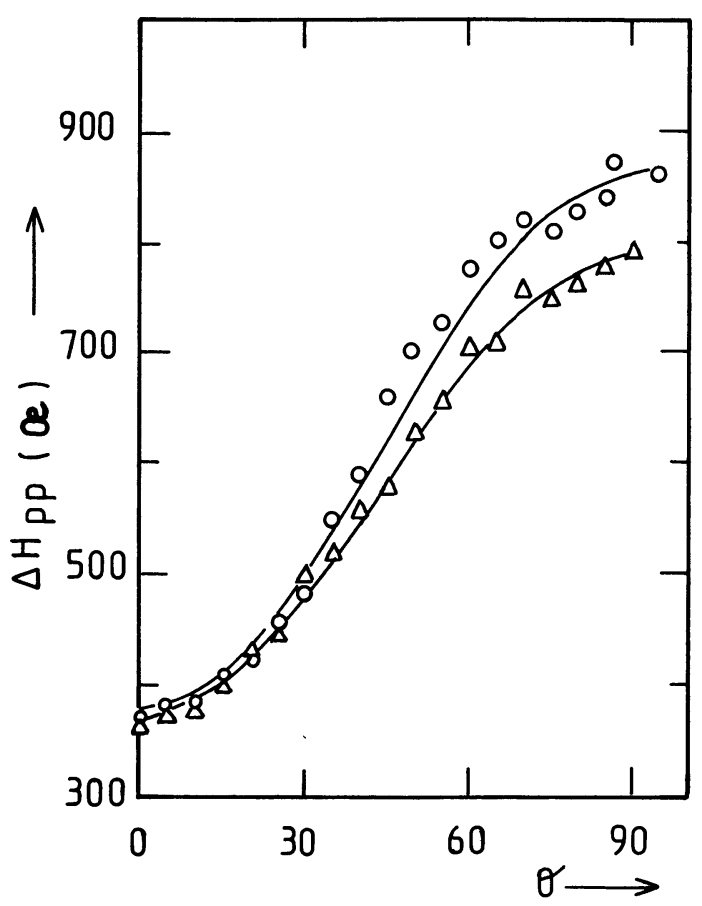

Fig. 2. - The ESR linewidth at room temperature in CHAC versus the angle $\theta$. The data are represented by circles when the magnetic field is placed in the $(b, c)$ plane and by triangles when the field is in the $(a, c)$ plane. The smooth lines have been obtained from a mean square adjustment of the functions :

$$
\begin{array}{lll}
\text { the upper curve } & \Delta H=788-413 \cos ^{2} \theta & (a, c) \text { plane } \\
\text { the lower curve } & \Delta H=860-485 \cos ^{2} \theta & (b, c) \text { plane }
\end{array}
$$

the results and conclusions drawn in TMMC cannot be applied to CHAC. In this compound, other interactions must be considered.

Groenendijk et al. [7] have extracted a value of the isotropic exchange : $J_{0} / k=70 \mathrm{~K}$ from their susceptibility data. In addition, they found a $1 \%$ Ising-like anisotropy. From heat capacity measurement, de Jonge et al. [8] found a lower value of $J_{0}(45 \mathrm{~K})$ with a $2 \%$ anisotropy.

Our own high temperature susceptibility measurement gives $J_{0} / k=47 \mathrm{~K}$ with an accuracy better than $10 \%$, in good agreement with the Eindhoven group value. It is highly probable that the anisotropic exchange interaction exceeds the dipolar interaction $\left(\omega_{\mathrm{D}}=\hbar \gamma e^{2} / \mathrm{r}^{3} \sim 10^{10} \mathrm{rad} . \mathrm{s}^{-1}\right.$, while $J_{0} / \hbar \sim 6 \times 10^{12} \mathrm{rad} . \mathrm{s}^{-1}$ ).

The Ising term behaves for the ESR linewidth just like the dipolar interaction, but here with an axial symmetry along the $b$-axis (as it can be inferred from the $g$-tensor). If the Ising term was predominant, one would obtain the same symmetry for the ESR spectrum and also a minimum of the linewidth in the $(b-c)$ plane, at about $\theta \sim 35^{\circ}$ (a new magic angle). However, cross terms involving dipolar and Ising interactions should break this symmetry, but the minimum in the $(b-c)$ plane should persist. This is not observed.

Another puzzling fact is the Lorentzian lineshape. It implies that a cut-off mechanism takes place for the diffusing modes larger (in frequency unit) than the linewidth. This cannot be due to the exchange coupling between chains because it is too weak $\left(\sim 10^{-3} J_{0}\right)$. So, another interaction has to be considered. One could think of the antisymmetric exchange interaction $\mathbf{d} . \mathbf{S}_{i} \mathbf{X S}_{j}$. It is known that $d$ can be potentially as large as $(\Delta g / g) J_{0}, \Delta g$ being the departure of the Lande 


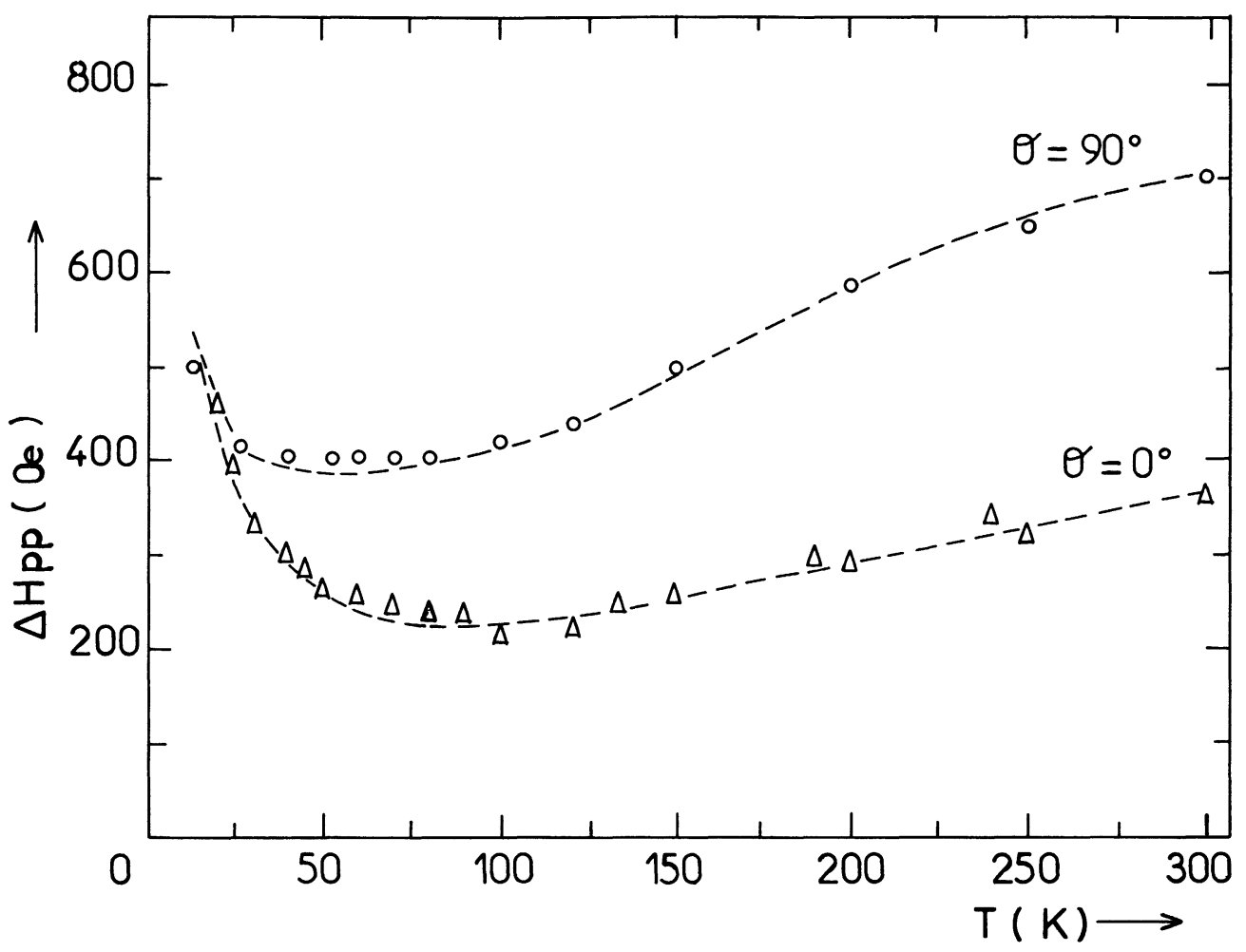

Fig. 3. - The ESR linewidth versus temperature for $\theta=0^{\circ}$ and $\theta=90^{\circ}$. The magnetic field is placed in the $(a, c)$ plane. The broken lines are only guides for the eyes.

factor from the value for the free electron [9]. Actually, its value is severly dependent on the crystal symmetry. For example, it vanishes when the two ions have a centre of inversion midway between them. Soos et al. $[10,11]$ have shown that the diffusive modes disappear when the ion sites are themselves centres of inversion. None of these symmetry conditions are strictly fulfilled here and the antisymmetric exchange (with $\mathbf{d} / / \mathrm{c}$ ) could explain satisfactorily our data. However, information concerning the presence of an antisymmetric exchange is hard to obtain from susceptibility measurement. In effect, such an interaction is not expected to influence static properties, as shown by Groenendijk et al. [7]. The surprising characteristics of the ESR in CHAC are probably related to a very strong coupling of the ions to the lattice. In TMMC, the electron spin-lattice relaxation occurs via a Bloembergen and Wang process. At high temperature, the relaxation time is very short but it becomes much longer at lower temperature. The situation is different in CHAC. Our measurements, using the modulation method [12], give extremely short times. The relaxation time is shorter for $\theta=0^{\circ}$ and its value is about $2 \times 10^{-10} \mathrm{~s}$ at $T=90 \mathrm{~K}$ (measurement at higher temperature could not be carried out). Clearly, it must contribute substantially to the linewidth at this orientation and at least partially at the others.

In a forthcoming publication we shall develop a more complete interpretation. 


\section{References}

[1] Steiner, M., Villain, J. and Windsor, C. G., Adv. Phys. 25 (1976) 87.

[2] Ablart, G., Bourdel, D., Pescia, J., Clement, S. and Renard, J. P., J. Physique-Lett. 42 (1981) L-219.

[3] Siegel, E., Ibrugger, J. and LagendiJk, A. J., J. Phys. C 15 (1982) 4583.

[4] JoH̀nson, J. D. and Bonner, J. C., Phys. Rev. Lett. 44 (1980) 616.

[5] Willett, R. D. and LandeE, C. P., J. Appl. Phys. 52 (1981) 2004.

[6] Schouten, J. C., van Der Geest, G. J., De Jonge, W. J. M. and Kopinga, K., Phys. Lett. 4 (1980) 1980.

[7] Groenendijk, H. A., Blote, H. W. J., van Duyneveldt, A. J., Gaura, R. M., Landee, C. P. and Willett, R. D., Physica 106B (1981) 47.

[8] Kopinga, K., Tinus, A. M. C. and De Jonge, W. J. M., Phys. Rev. B 25 (1982) 4685.

[9] MoriYa, T., Phys. Rev. 120 (1960) 91.

[10] Soos, Z. G., Cheung, T. T. P. and McGregor, K. T., Chem. Phys. Lett. 3 (1977) 600.

[11] Soos, Z. G., McGregor, K. T., Cheung, T. T. P. and Silverstein, A. J., Phys. Rev. B 16 (1977) 3036.

[12] Ablart, G. and Pescia, J., Phys. Rev. B 22 (1980) 1150. 